\title{
Increased Risk of Intraventricular Hemorrhage in Preterm Infants with Thrombophilia
}

\author{
JARI PETÄJÄ, LEENA HILTUNEN, AND VINETA FELLMAN \\ Hospital for Children and Adolescents, University of Helsinki, Stenbäckinkatu 11, FIN-00290 Helsinki, \\ Finland [J.P., V.F.]; and The Finnish Red Cross Blood Transfusion Service, Kivihaantie 7, FIN-00310 \\ Helsinki, Finland [L.H.]
}

\begin{abstract}
ABST
The multifactorial etiology of cerebral intraventricular hem-
orrhage (IVH) may involve coagulation disturbances and venous
infarction. We tested whether coagulation abnormalities associ-
ated with adult venous thrombosis would constitute a risk factor
for IVH in newborn infants. In 22 infants (gestational age
$24.3-39.9$ wk, median 28.0 wk) with neonatal IVH grade II to
IV, the frequencies of congenital resistance to activated protein C
due to a point mutation in the factor V gene (Gln506-FV) and a
polymorphism in the prothrombin gene (G20210A-FII) were
assessed and compared with those observed in 29 premature
newborn infants without IVH and in 302 (Gln506-FV) or 526
(G20210A-FII) healthy adults. In infants with IVH, four (18\%)
heterozygous carriers of Gln506-FV and one (5\%) heterozygous
carrier of G20210A-FII were found. One infant without IVH was
heterozygous for Gln506-FV ( $3 \%$ ). When compared with the
frequency of Gln506-FV in the general population, the odds ratio
for being a carrier of Gln506-FV for patients with IVH was 5.9
(95\% confidence interval $1.7-20.3, p=0.013)$ and for patients
without IVH 0.9 (95\% confidence interval $0.1-7.6, p>0.99)$.
\end{abstract}
The absolute risk of IVH in a newborn infant with heterozygous Gln506-FV and born before $30 \mathrm{wk}$ of gestation was estimated at $80 \%$, whereas the corresponding risk for all infants born before 30 wk was $14 \%$. Gln506-FV was more common in newborn infants with IVH than in the general population, whereas there was no difference in the frequencies of Gln506-FV in infants without IVH and in the general population. Thus, Gln506-FV may be a risk factor of IVH. The risk of IVH in a premature infant with Gln506-FV or other established thrombophilic coagulation abnormality may be considerable. (Pediatr Res 49: 643646, 2001)

Abbreviations
GIn506-FV, mutated factor V with arginine506 to glutamine
substitution (factor V Leiden)
G20210A-FII, prothrombin G20210A polymorphism
IVH, intraventricular hemorrhage
PVL, periventricular leukomalacia

IVH is a major cause of morbidity and mortality in premature infants currently affecting up to $20 \%$ of those infants weighing less than $1500 \mathrm{~g}$ (1). The etiology of IVH remains undefined but includes multiple factors affecting blood flow and perfusion pressure in the periventricular area. However, developmental structural factors are crucial for the initial IVH appearing in the subependymal germinal matrix in which vessel walls are without collagen or smooth muscle cell support (2). The primary event in a germinal matrix hemorrhage may be a simple mechanical rupture of the immature developmental vessels unable to tolerate fluctuations in intraluminal pressure or the hemorrhage may be secondary to reperfusionassociated vascular injury. On the other hand, stasis produced by venous obstruction and cerebral venous infarction are con-

Received June 27, 2000; accepted December 12, 2000.

Correspondence: Jari Petäjä, M.D., Ph.D., Hospital for Children and Adolescents, University of Helsinki, Stenbäckinkatu 11, FIN-00290 Helsinki, Finland; e-mail: jari.petaja@dlc.fi sidered the probable pathogenic mechanisms for parenchymal hemorrhage associated with IVH (3-6).

Understanding of the pathogenesis of venous thrombosis has improved, as several congenital hemostatic abnormalities have been shown to increase the risks of juvenile and familial venous thrombosis (7). A point mutation in the factor $\mathrm{V}$ gene resulting in the arginine506 to glutamine substitution in factor $\mathrm{V}$ (Gln506-FV) and the G20210A polymorphism in the prothrombin gene are common prothrombotic genetic abnormalities in Caucasian populations $(8,9)$. However, the clinical phenotype of these genetic abnormalities in newborn infants remains partially undefined. Logical candidate disease states include all situations associated with the disturbed hemostatic system in which thrombosis as a pathogenic mechanism has not been excluded. Accordingly, during the perinatal period, congenital thrombophilic risk factors have been associated with obstetric complications partially related to placental infarction $(10,11)$ as well as with arterial and venous thrombotic complications in newborn and older infants (12-18). A recent 
report of eight patients with hydrocephalus suggested that Gln506-FV might be associated also with IVH (19). In the current prospective study, the hypothesis that Gln506-FV or other thrombophilic abnormalities may predispose to IVH was tested.

\section{METHODS}

Patients. The present study was conducted at the neonatal intensive care unit of the Hospital for Children and Adolescents, University of Helsinki. Seven hundred five patients admitted between January 1, 1998 and September 10, 1999 were eligible for the study. Routine cranial sonography was performed on infants born before $32 \mathrm{wk}$ of gestation on the first day of life and was repeated at least once during the first week and thereafter at 2 and at 4-6 wk of age. For infants born at or after $32 \mathrm{wk}$ of gestation, the cranial sonography was performed at least once during the first week of life. Additional scans were performed on clinical indications.

Based on the criteria of Papile et al. (20), 28 patients developed a grade II to IV IVH $(4.0 \%)$. From these patients, informed parental consent for blood samples was obtained for 22 patients (infants with IVH). Twenty-nine newborn infants who had no IVH and were of similar gestational age and had similar obstetric background as the patients with IVH were enrolled as controls (control infants). The control infants were enrolled consecutively between February 1999 and September 1999. Selected clinical information of the patients with and without IVH are given in Table 1. Patients with grade I IVH were not enrolled as either patients or controls.

Healthy subjects. The prevalence of Gln506-FV [3.6\%, 95\% confidence interval (CI) $1.8-6.4 \%$ ] was obtained by studying a random population sample of 302 healthy young men. The prevalence of G20210A-FII $(1.1 \%, 95 \%$ CI $0.4-2.5 \%)$ was investigated in a random group of 526 healthy adults. Control materials were studied at the Department of Hemostasis in the Finnish Red Cross Blood Transfusion Service.

Samples and laboratory methods. In the infants with IVH, the sample volume was dependent on the weight of the patient. A blood sample of $0.9,1.8$, or $2.7 \mathrm{~mL}$ of blood was drawn from infants weighing less than $1000,1000-2000$, or over $2000 \mathrm{~g}$, respectively. Nine parts of blood were collected in one part of $0.109 \mathrm{M}$ trisodium citrate and immediately transferred to the laboratory of hemostasis at the University Hospital of Helsinki, where platelet-poor plasma was separated by centrifugation and subsequently assayed for protein $\mathrm{C}$ activity (PC), antithrombin activity (AT), free protein $\mathrm{S}$ antigen (PS), thrombin time, and presence of lupus anticoagulant and anticardiolipin antibodies. Published normal ranges for PC, AT, and PS were used to suggest or exclude congenital deficiencies of these anticoagulants (21). After isolation of genomic DNA from leukocytes, the presence of Gln506-FV and G20210A-FII was detected essentially as described $(8,22)$. In the control infants, only genomic assays for Gln506-FV and G20210A-FII were performed.

Statistical methods. The Mann Whitney $U$ and Fisher's exact tests were used to compare clinical characteristics of the control infants and infants with IVH. The differences in the frequencies of Gln506-FV and G20210A-FII between the different groups were compared using the Fisher's exact test. The odds ratios (OR) with associated $\mathrm{CI}$ were calculated.

Ethics. The present study was approved by the ethics committee of the Hospital for Children and Adolescents and, for the part of studies on healthy adults, by the ethics committee of the Finnish Red Cross Blood Transfusion Service. Informed parental consent was obtained for each patient before entry into the study.

\section{RESULTS}

The incidence of IVH decreased as a function of increasing gestational age (Table 2). From the 28 patients with IVH, 22 could be enrolled to the study, and the following parameters were determined: Gln506-FV in 22, G20210A-FII in 21, anticardiolipin antibodies in 19, PC in 18, AT in 18, PS in 17, lupus anticoagulant in 10 , and thrombin time in eight. The reason for not carrying out all plasma-based assays in all patients was the limited amount of plasma available, as the volume of the blood sample was based on the weight of the patient (see "Methods"). The following abnormal results were found (Table 3): heterozygosity for Gln506-FV in four (18\%), heterozygosity for G20210A-FII in one (5\%), PC deficiency in one ( $6 \%$ of the tested), and lupus anticoagulant in one $(10 \%$ of the tested). No patient had more than one abnormal result. In the patient sample with lupus anticoagulant, no anticardiolipin antibodies were found. The presence of lupus anticoagulant

Table 1. Clinical characteristics of patients with and without IVH

\begin{tabular}{|c|c|c|c|}
\hline & Controls & $\begin{array}{l}\text { Patients with } \\
\text { grade II-IV IVH }\end{array}$ & $p$ \\
\hline$n$ & 29 & 22 & \\
\hline Male/female & $20 / 9$ & $12 / 10$ & 0.38 \\
\hline Birth weight (g, median and range) & $1050(450-2520)$ & $1090(500-2920)$ & 0.68 \\
\hline \multicolumn{4}{|l|}{ Cause of prematurity* } \\
\hline Premature rupture of membranes & 11 & 6 & 0.55 \\
\hline Placental abruption & 0 & 2 & 0.18 \\
\hline Other & 7 & 3 & 0.48 \\
\hline
\end{tabular}

Mann Whitney $U$ test (gestational age and birth weight) and Fisher's exact test were used to calculate differences between groups. $*$ Two patients with IVH were born at term. 
Table 2. Incidence of IVH during the study period

\begin{tabular}{lcccc}
\hline \multicolumn{5}{c}{ Patients } \\
GA & $\begin{array}{c}\text { Patients } \\
\text { with IVH } \\
(n)\end{array}$ & $\begin{array}{c}\text { IVH } \\
\text { (n) }\end{array}$ & $\begin{array}{c}\text { Absolute risk of } \\
\text { IVH in carriers } \\
\text { of Gln506-FV } \\
(\%)\end{array}$ \\
\hline $23-26$ & 50 & 10 & $10(20 \%)$ & $100(34-100)$ \\
27 & 67 & 2 & $12(18 \%)$ & $100(30-100)$ \\
28 & 94 & 1 & $13(14 \%)$ & $82(24-100)$ \\
29 & 132 & 5 & $18(14 \%)$ & $80(23-100)$ \\
30 & 179 & 1 & $19(11 \%)$ & $63(18-100)$ \\
31 & 217 & 1 & $20(9 \%)$ & $54(16-100)$ \\
32 & 266 & 1 & $21(8 \%)$ & $47(13-100)$ \\
33 & 316 & 2 & $23(7 \%)$ & $43(12-100)$ \\
34 & 356 & 2 & $25(7 \%)$ & $41(12-100)$ \\
35 & 398 & 0 & $25(6 \%)$ & $37(11-100)$ \\
36 & 432 & 1 & $26(6 \%)$ & $36(10-100)$ \\
$>36$ & 705 & 2 & $28(4 \%)$ & $23(7-81)$ \\
\hline
\end{tabular}

Patients with IVH $=$ the incidence of at least grade II IVH among the 705 patients treated in the neonatal intensive care unit of Hospital for Children and Adolescents during the study period. IVH cumulative $=$ infants with IVH presented as cumulative numbers up to that gestational age (GA). Estimates of absolute risk of IVH in heterozygous carriers of Gln506-FV were calculated by multiplying the incidence of IVH by the OR of 5.9 for heterozygous Gln506-FV in patients with IVH compared with Gln506-FV in healthy adults (Table 4). The range of absolute risk was estimated by using the corresponding 95\% CI (1.7-20.3) (Table 4).

was demonstrated by prolonged Russell's viper venom time and activated partial thromboplastin time, both insensitive to sample dilution in association with a normal thrombin time. The patient with PC deficiency had the PC level measured twice, at $1 \mathrm{~d}$ (gestational age $27+5$ wk) and at $40 \mathrm{~d}$ (gestational age $33+3 \mathrm{wk}$ ) of age, and the values were 12 and $10 \%$ of the adult mean, respectively. Both of the parents were found to have normal levels of protein C. Thus, the patient was considered to have PC deficiency based on the published normal range for PC (21). The defect could be a congenital one due to a new mutation or an acquired one. The levels of both AT ( $37 \%$ of the adult mean) and PS ( $66 \%$ of the adult mean) were much higher and normal for age. Overall, the combined frequency of established thrombophilic abnormalities in the infants with IVH was 7/22 (32\%).

In the 29 control infants, only Gln506-FV and G20210A-FII were studied, and one patient was found to be heterozygous for Gln506-FV (3\%).

The frequencies of Gln506-FV and G20210A-FII in patients with IVH, in patients without it, and in healthy adults were compared (Table 4). OR for being a carrier of Gln506-FV for patients with IVH was 5.9 when compared with healthy adults $(p=0.013)$. Very similar albeit statistically not significant, OR of 6.2 for being a carrier of Gln506-FV was observed when patients with IVH were compared with premature infants without IVH. For this comparison, OR further increased to 8.2 when heterozygosity for G20210A-FII was also included in the analysis $(p=0.073)$ (Table 4).

In the whole patient series, the risk of a grade II to IV IVH was $14 \%$ for infants born before $30 \mathrm{wk}$ of gestation and $2 \%$ for those born at $30 \mathrm{wk}$ or later (Table 2). The corresponding estimated risks of IVH in subjects who are heterozygous for Gln506-FV were 80 and $10 \%$, respectively (Table 2).

Occurrence of PVL in the patients with IVH was also analyzed in relation to the thrombophilic abnormalities. Four of the seven patients (57\%) with a thrombophilic abnormality had evidence of PVL, whereas the same was true for eight of the 15 patients $(53 \%)$ with IVH but no recognized thrombophilia (a nonsignificant difference).

\section{DISCUSSION}

Heterozygosity for Gln506-FV was found in $18 \%$ of patients with IVH, whereas it was present in only $3 \%$ of infants without IVH and in $4 \%$ of healthy adults in a large population sample. This suggests that Gln506-FV may be a risk factor for neonatal IVH. The fact that in three out of four patients with Gln506$\mathrm{FV}$, the IVH was considered to have occurred already antenatally (Table 4) also supports a causal role for Gln506-FV in IVH. Also of note is the gestational age $(38+2 \mathrm{wk})$ of one patient with Gln506-FV and grade IV IVH. It is plausible to suggest that the otherwise very low risk of IVH near term was aggravated by Gln506-FV in this particular patient. Overall, the current findings are in good agreement with the study in which eight children with hydrocephalus were investigated and three carriers of Gln506-FV were found (19).

In addition to the presence of Gln506-FV, however, three other established thrombotic risk factors, namely heterozygosity of G20210A-FII, lupus anticoagulant, and protein C deficiency, were found among the infants with IVH. These additional findings increase the prevalence of thrombophilic abnormalities to $32 \%$ among patients with IVH and thus suggest that not only Gln506-FV but also thrombophilia as a whole may be common in newborn infants with IVH. From the viewpoint of pathogenic mechanisms, this finding supports the concept that venous infarction may be involved in the development and/or progression of IVH (3-6). The overall role of prothrombotic coagulation disturbances may even be more

Table 3. Clinical data on the seven patients with IVH and a thrombophilic coagulation abnormality

\begin{tabular}{lllll}
\hline $\begin{array}{c}\text { Patient and cause of } \\
\text { prematurity }\end{array}$ & Sex / GA / BW & Hemostatic abnormality & IVH & Additional risk factors \\
\hline 1. Preeclampsia & $\mathrm{M} / 27+5 / 879$ & Protein C deficiency & IV & Intrauterine bleed \\
2. Preeclampsia & $\mathrm{M} / 29+0 / 1100$ & Lupus anticoagulant & III & Intrauterine bleed \\
3. PROM & $\mathrm{M} / 29+6 / 1480$ & G20210A-FII & II & None \\
4. Placental abruption & $\mathrm{F} / 29+0 / 1285$ & Gln506-FV & II & None/intrauterine bleed \\
5. PROM & $\mathrm{F} / 30+5 / 1450$ & Gln506-FV & III* & E. coli meningitis \\
6. Maternal varicella & $\mathrm{M} / 31+0 / 1980$ & Gln506-FV & III* & Maternal varicella/intrauterine bleed \\
7. Term & $\mathrm{M} / 38+2 / 2920$ & Gln506-FV & IV & Intrauterine bleed \\
\hline
\end{tabular}

GA indicates gestational age (wk); BW, birth weight (g); PROM, premature rupture of membranes. In the five patients with intrauterine IVH, the timing was based on the clinical features of the child and the first postnatal ultrasonography. *bilateral. 
Table 4. Comparison of frequencies of Gln506-FV and G20210A-FII between the different study groups

\begin{tabular}{llll}
\hline & $\begin{array}{c}\text { Frequency of } \\
\text { the defect }\end{array}$ & OR $(95 \% \mathrm{CI})$ & \multicolumn{1}{c}{$p$} \\
\hline Gln506-FV & & & \\
IVH $v s$ healthy adults & $4 / 22 v s 11 / 302$ & $5.9(1.7-20.3)$ & 0.013 \\
IVH $v$ no IVH & $4 / 22 v s 1 / 29$ & $6.2(0.6-59.9)$ & 0.15 \\
No IVH $v$ h healthy adults & $1 / 29 v s 11 / 302$ & $0.9(0.1-7.6)$ & $>0.99$ \\
G20210A-FII & & & \\
IVH $v$ s healthy adults & $1 / 22 v s 6 / 526$ & $4.1(0.5-35.4)$ & 0.25 \\
IVH $v s$ no IVH & $1 / 22 v s 0 / 29$ & - & 0.43 \\
No IVH $v$ healthy adults & $0 / 29 v s 6 / 526$ & - & $>0.99$ \\
Gln506-FV + G20210A-FII & & & \\
IVH $v s$ no IVH & $5 / 22 v s 1 / 29$ & $8.2(0.9-76.6)$ & 0.073
\end{tabular}

The $p$ values were calculated with Fisher's exact test.

pronounced than suggested by the current findings because sick newborn infants most often have disseminated activation of coagulation with associated short-lived secondary deficiencies of physiologic anticoagulants $(23,24)$. Further, because not all plasma-based assays could be carried out in all patients, the combined $32 \%$ prevalence of thrombophilia may be regarded as a low estimate.

We suggest that IVH is a new candidate for the list of disease states that may be triggered by thrombophilic coagulation abnormalities and especially by Gln506-FV. The primary clinical phenotype of the various congenital defects of physiologic anticoagulation (AT, PC, and PS deficiencies as well as Gln506-FV) and G20210A-FII undoubtedly is an increased risk for venous but not arterial thromboembolic disease starting in early adulthood (7). For unknown reasons, during childhood, the carriers of these defects show remarkably low thrombotic risk unless copredisposed to strong acquired thrombotic risk factors like deep vein catheters or malignancy (25, 26). In contrast, during the neonatal period, thrombophilia seems to be associated with complicated multiple clinical risks including venous catheter-triggered thrombi (24-27), arterial stroke $(12-14,16-18)$, and, now, IVH (19). However, in premature infants, the general incidence of large venous thrombi as well as arterial stroke is very low compared with the relatively frequent occurrence of IVH. Thus, our data are novel in suggesting that in very premature newborn infants, the most likely thrombophilia-associated adverse clinical event could be IVH.

Acknowledgments. The authors thank Kaija Aspholm and Anne Salonen for technical help throughout the study.

\section{REFERENCES}

1. Roland EH, Hill A 1997 Intraventricular hemorrhage and posthemorrhagic hydrocephalus. Clin Perinatol 24:589-605
2. Kuban KCK, Gilles FH 1985 Human telencephalic angiogenesis. Ann Neurol 17:539-548

3. Volpe JJ 1997 Brain injury in the premature infant. Clin Perinatol 24:567-587

4. Takashima S, Mito T, Ando Y 1986 Pathogenesis of periventricular white matter hemorrhages in preterm infants. Brain Dev 8:25-30

5. Gould SJ, Howard S, Hope PL, Reynolds EO 1987 Periventricular intraparenchymal cerebral haemorrhage in preterm infants: the role of venous infarction. J Pathol 151:197-202

6. Taylor GA 1995 Effect of germinal matrix hemorrhage on terminal vein position and patency. Pediatr Radiol 25:37-40

7. Martinelli I, Mannucci PM, De Stefano V, Taioli E, Rossi V, Crosti F, Paciaroni K, Leone G, Faioni EM 1998 Different risks of thrombosis in four coagulation defects associated with inherited thrombophilia. A study of 150 families. Blood 92:23532358

8. Poort SR, Rosendaal FR, Reitsma PH, Bertina RM 1996 A common genetic variation in the 3 -untranslated region of the prothrombin gene is associated with elevated plasma prothrombin levels and an increase in venous thrombosis. Blood 88:36983703

9. Petäjä J, Griffin JH 1997 Activated protein C resistance. What have we learned now that the dust has settled? Ann Med 29:469-472

10. Tormenen D, Simioni P, Prandoni P, Luni S, Innella B, Sabbion P, Girolami A 1999 The risk of fetal loss in family members of probands with factor V Leiden mutation. Thromb Haemost 82:1237-1239

11. Kupferminc MJ, Eldor A, Steinman N, Many A, Bar-Am A, Jaffa A, Fait G, Lessing JB 1999 Increased frequency of genetic thrombophilia in women with complications of pregnancy. N Engl J Med 340:9-13

12. Hagstrom JN, Walter J, Bluebond-Langner R, Amatniek JC, Manno CS, High KA 1998 Prevalence of the factor V Leiden mutation in children and neonates with thromboembolic disease. J Pediatr 133:777-781

13. Bonduel M, Sciuccati G, Hepner M, Torres AF, Pieroni G, Frontroth JP 1999 Prethrombotic disorders in children with arterial ischemic stroke and sinovenous thrombosis. Arch Neurol 56:967-971

14. Nowak-Göttl U, Sträter R, Dübbers A, Oleszuk-Raschke K, Vielhaber H 1996 Ischaemic stroke in infancy and childhood: role of the Arg506 to Gln mutation in the factor $\mathrm{V}$ gene. Blood Coagul Fibrinolysis 7:684-688

15. deVeber G, Mongale P, Chan A, MacGregor D, Curtis R, Lee S, Vegh P, Adams M, Marzinotto V, Leaker M, Massicotte MP, Lillicrap D, Andrew M 1998 Prothrombotic disorders in infants and children with cerebral thromboembolism. Arch Neurol 55:1539-1543

16. Zenz W, Bodo Z, Plotho J, Streif W, Male C, Bernert G, Rauter L, Ebetsberger G, Kaltenbrunner K, Kurnik P, Lischka A, Paky F, Ploier R, Hofler G, Mannhalter C, Muntean W 1998 Factor V Leiden and prothrombin gene G 20210 A variant in children with ischemic stroke. Thromb Haemost 80:763-766

17. Becker S, Heller C, Gropp F, Scharrer I, Kreuz W 1998 Thrombophilic disorders in children with cerebral infarction. Lancet 352:1756-1757

18. Nowak-Göttl U, Koch HG, Aschka I, Kohlhase B, Vielhaber H, Kurlemann G, Oleszuck-Raschke K, Kehl HG, Jurgens H, Schneppenheim R 1996 Resistance to activated protein $\mathrm{C}$ (APCR) in children with venous or arterial thromboembolism. Br J Haematol 92:992-998

19. Riikonen R, Kekomäki R 1998 Resistance to activated protein C (APC) in childhood hydrocephalus. Thromb Haemost 79:1059-1060

20. Papile LA, Burstein J, Burstein R, Koffler H 1978 Incidence and evolution of subependymal and intraventricular hemorrhage: a study of infants with birth weights less than 1,500 gm. J Pediatr 92:529-534

21. Andrew M, Paes B, Johnston M 1990 Development of the hemostatic system in the neonate and young infant. Am J Pediatr Hematol Oncol 12:95-104

22. Bertina RM, Koelman BP, Koster T, Rosendaal FR, Dirven RJ, De Ronde H, Van Der Velden PA, Reitsma PH 1996 Mutation in blood coagulation factor V associated with resistance to activated protein $\mathrm{C}$. Nature 369:64-67

23. Schmidt B, Vegh P, Johnston M, Andrew M, Weitz J 1993 Do coagulation screening tests detect increased generation of thrombin and plasmin in sick newborn infants? Thromb Haemost 69:418-421

24. Petäjä J, Peltola K, Sairanen H, Leijala M, Kekomäki R, Vahtera E, Siimes MA 1996 Fibrinolysis, antithrombin III, and protein C in neonates during open heart surgery. J Thorac Cardiovasc Surg 112:665-671

25. Andrew M, David M, Adams M, Ali K, Anderson R, Barnard D, Bernstein M, Brisson L, Cairney B, DeSai D, Grant R, Israels S, Jardine L, Luke B, Massicotte P, Silva M 1994 Venous thromboembolic complications (VTE) in children: first analyses of the Canadian registry of VTE. Blood 83:1251-1257

26. Nowak-Göttl U, Dübbers A, Kececioglu D, Koch HG, Kotthoff S, Runde J, Vielhaber H 1997 Factor V Leiden, protein C, and lipoprotein (a) in catheter-related thrombosis in childhood. A prospective study. J Pediatr 131:608-612

27. Petäjä J, Lundström U, Sairanen H, Marttinen E, Griffin JH 1996 Central venous thrombosis after cardiac surgery in children. J Thorac Cardiovasc Surg 12:883889 